\title{
IUCrJ
}

Volume 8 (2021)

Supporting information for article:

High-resolution cryo-EM using beam-image shift at $200 \mathrm{keV}$ Jennifer N. Cash, Sarah Kearns, Yilai Li and Michael A. Cianfrocco 
IUCrJ (2021). 8, doi:10.1107/S2052252520013482

Supporting information, sup-1

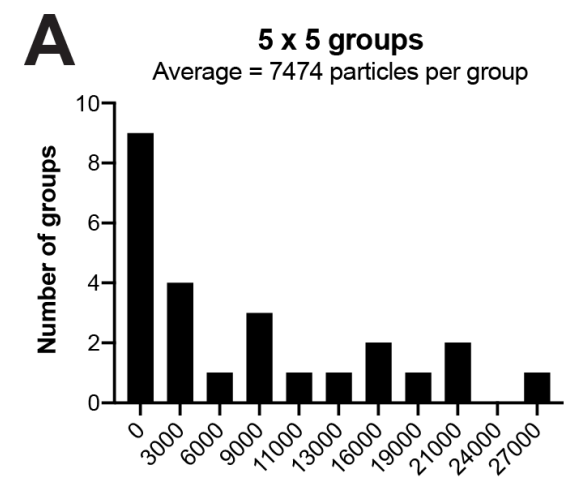

Particles per group

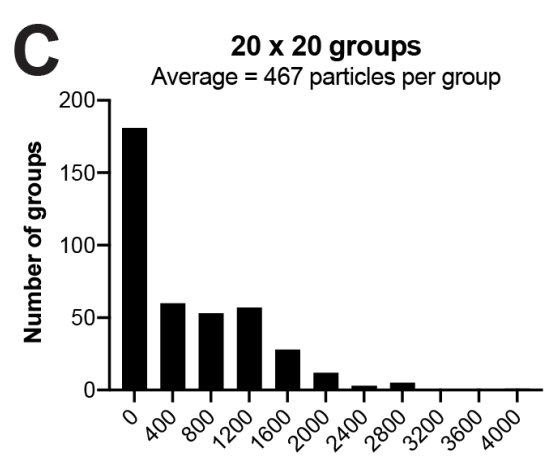

Particles per group

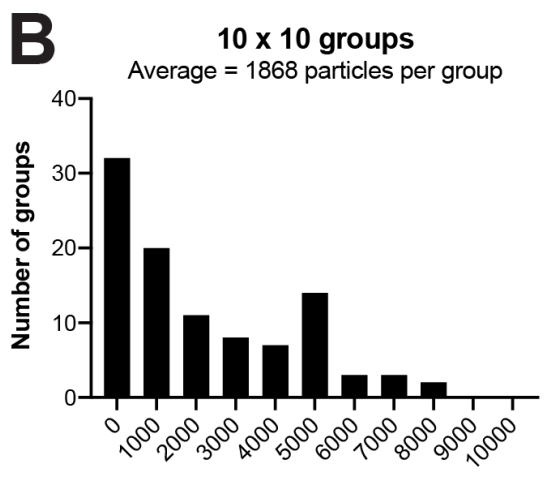

Particles per group

Supplemental Figure 1 - Particle numbers per group. Histograms of particle distribution per beam tilt grouping. (A) $5 \times 5$, (B) $10 \times 10$, (C) $20 \times 20$. 


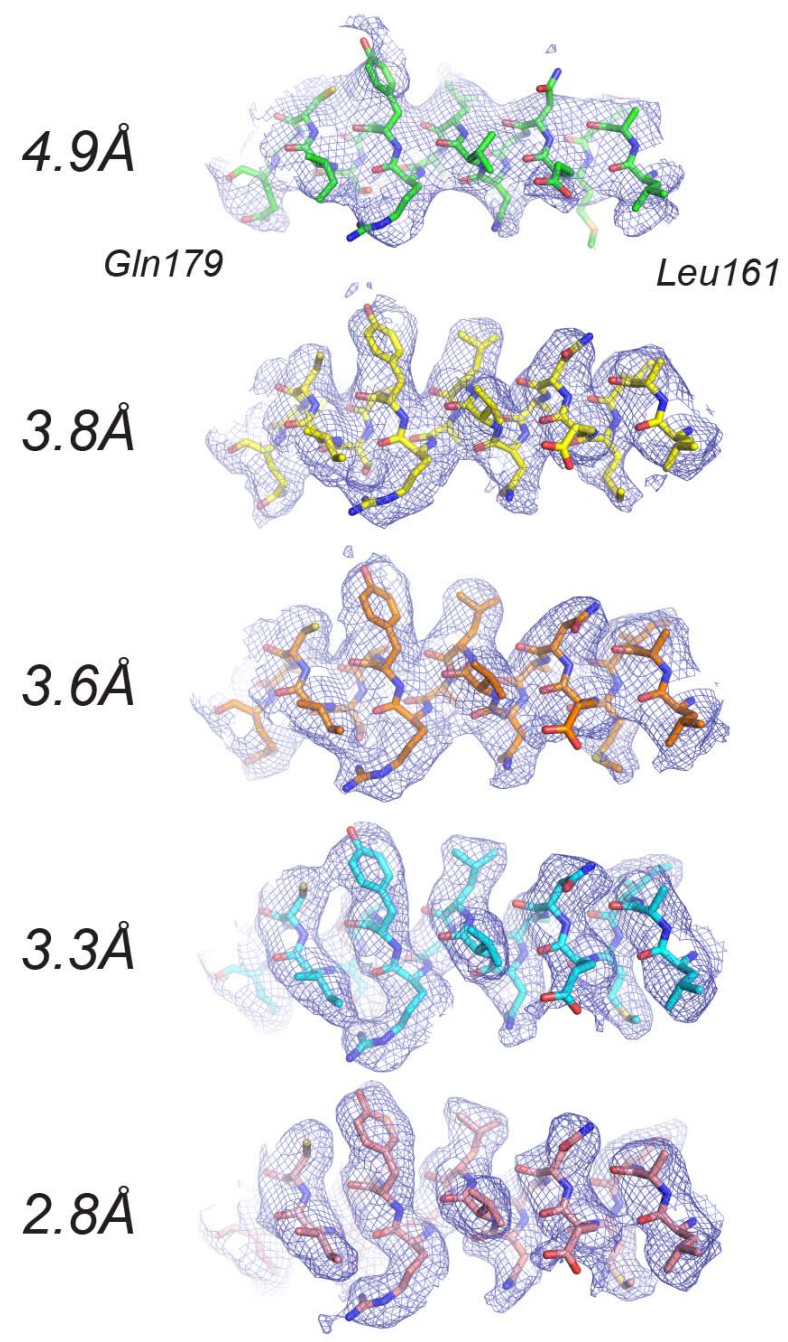

Supplemental Figure 2 - Representative densities from iterative beam-tilt refinements. Sharpened densities with associated models highlight changes in density quality through iterative rounds of beam-tilt refinement corresponding to structures from Figure 4A-4E. 

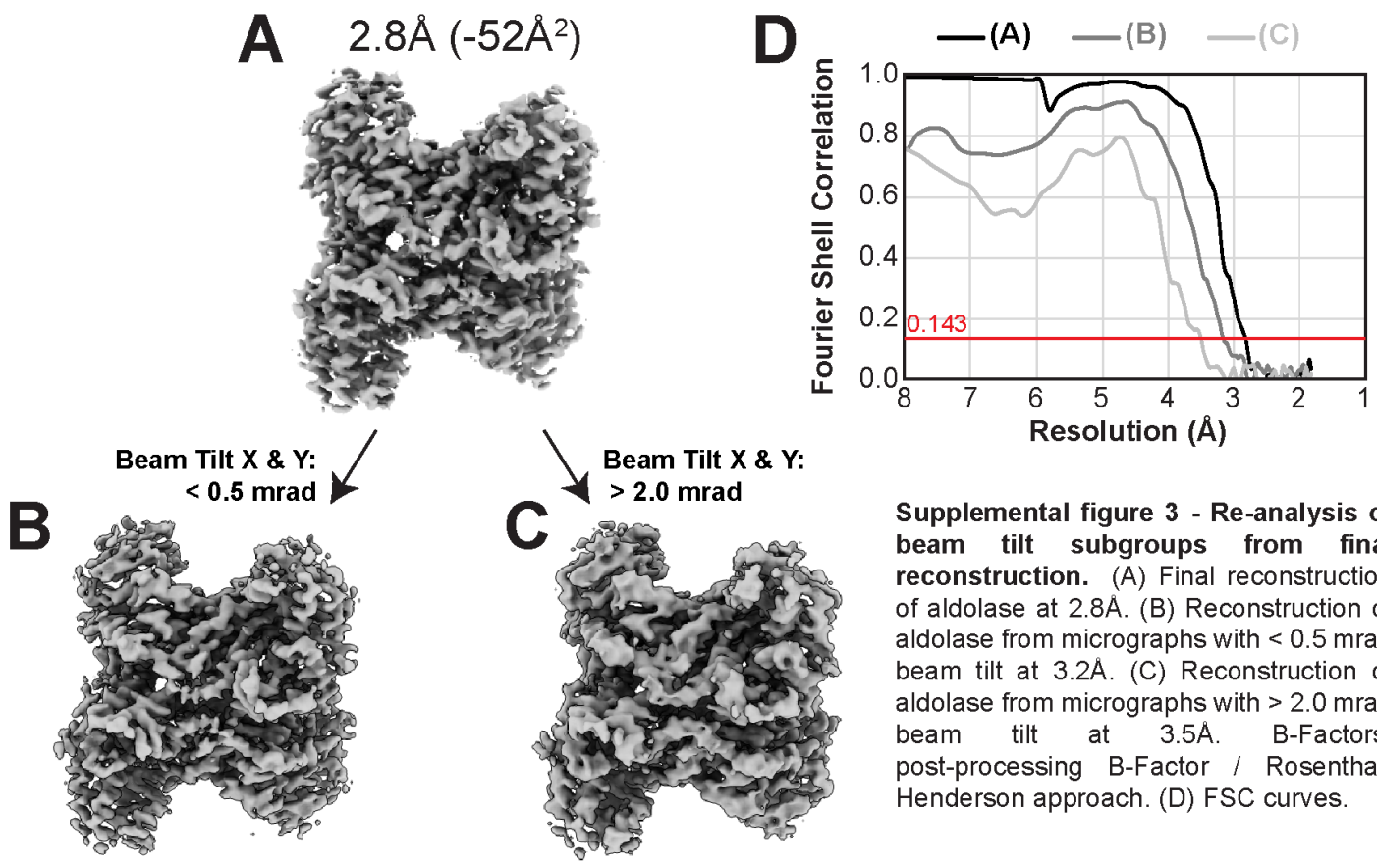

Supplemental figure 3 - Re-analysis of beam tilt subgroups from final reconstruction. (A) Final reconstruction of aldolase at $2.8 \AA$. (B) Reconstruction of aldolase from micrographs with $<0.5 \mathrm{mrad}$ beam tilt at $3.2 A$. (C) Reconstruction of aldolase from micrographs with $>2.0 \mathrm{mrad}$ beam tilt at 3.5A. B-Factors: post-processing B-Factor / RosenthalHenderson approach. (D) FSC curves. 
A Map to Model FSC

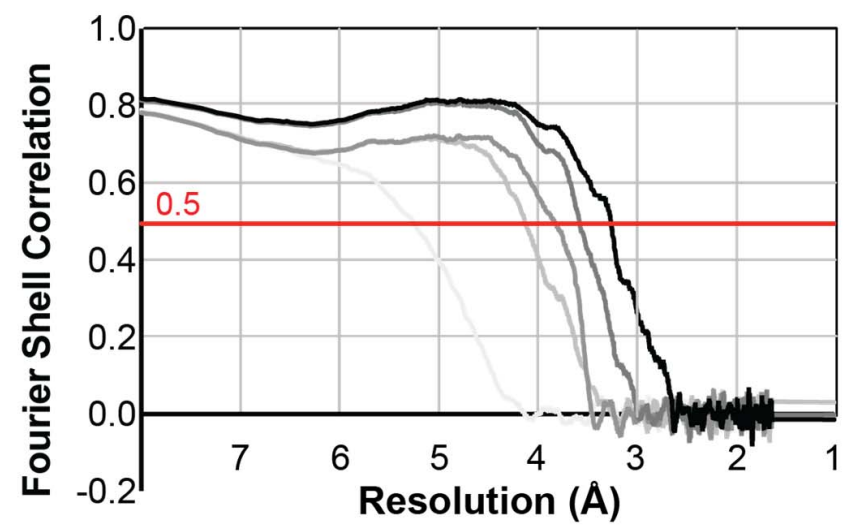

Fig. $4 \mathrm{E}(2.8 \AA)$

- Fig. $4 \mathrm{D}(3.3 \AA)$

- Fig. $4 \mathrm{C}(3.6 \AA)$

Fig. 4B $(3.8 \AA)$

Fig. $4 \mathrm{~A}(4.9 \AA)$

B

\section{B-factor plots}

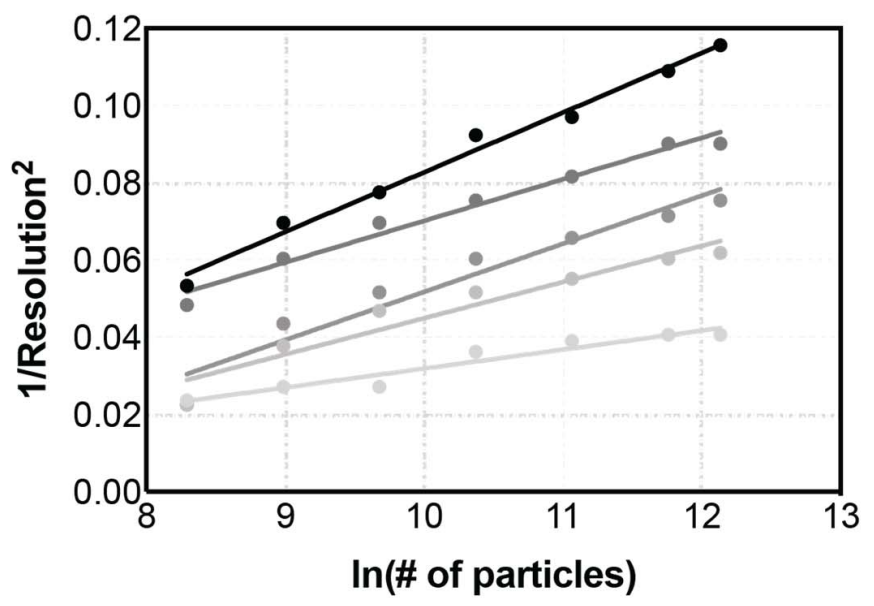

$\rightarrow 2.8 \AA(B=-129.9)$

$\rightarrow 3.3 \AA \AA(B=-185.1)$

$\multimap 3.6 \AA(B=-202.0)$

$-3.8 \AA(B=-274.0)$ $4.9 \AA(B=-400.0)$

Supplemental figure 4 - Structure validation during data analysis.

(A) Map-to-model FSC comparing our maps from Fig. 4A-E with $5 \mathrm{vy} 5$ as the model. (B) Rosenthal-Henderson plots showing particle number and resolution to determine B-factors for each reconstruction. 
A

B
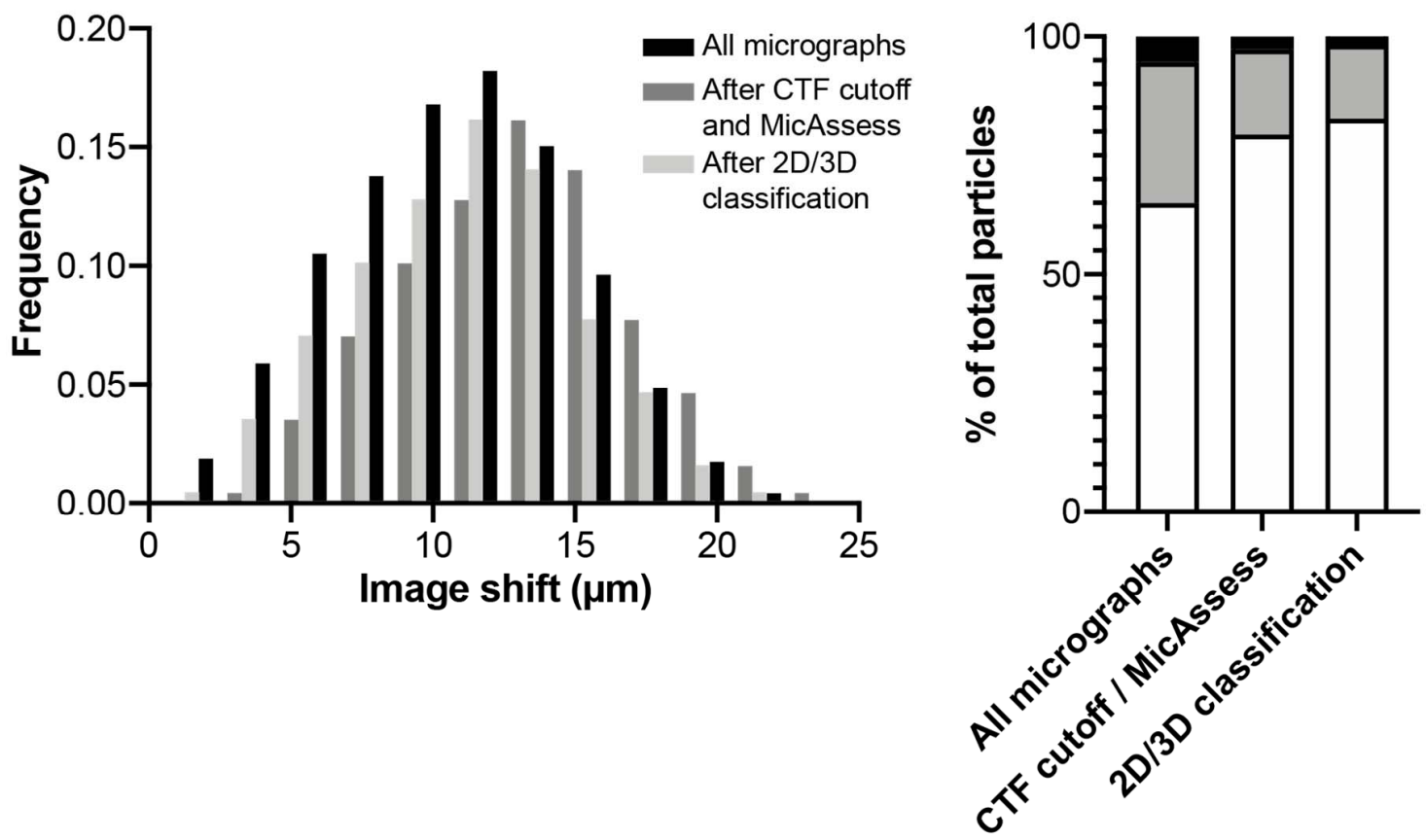

Defocus value

$>2.0 \mathrm{um}$

$1.5<\mathrm{x}<2$ um $\square<1.5$ um

Supplemental figure 5 - Quantification of micrograph image shift and particle defocus. (A) For all micrographs from the dataset (black bars), the distance from $(0,0)$ was determined and plotted as a function of frequency. The same was done for the subset of micrographs kept after CTF cut off and MicAsses (dark grey bars) and 2D/3D classification (light grey bars). (B) For every particle extracted, the defocus was binned into lower than 1.5 um (white), higher than 2 um (black), or inbetween (grey). The same was done for the subset if particles kept after CTFcutoff and MicAssess $(500,001)$ and 2D/3D classification (186,471 particles). 
IUCrJ (2021). 8, doi:10.1107/S2052252520013482 Supporting information, sup-6 Supplemental Table S1 - Cryo-EM data collection, refinement, and validation statistics.

\begin{tabular}{|c|c|}
\hline & Aldolase (19apr12a) \\
\hline Microscope & Talos-Arctica \\
\hline Detector & Gatan K2 \\
\hline Voltage (keV) & 200 \\
\hline Electron exposure $\left(\mathrm{e}^{-} / \AA^{2}\right)$ & 43 \\
\hline Defocus range $(\mu \mathrm{m})$ & $0.8-2$ \\
\hline Data collection mode & Beam-image shift \\
\hline Micrographs collected (per hour) & 73 \\
\hline Original pixel size (Å) & 0.91 \\
\hline Symmetry imposed & D2 \\
\hline Initial number of micrographs & 2,111 \\
\hline Final number of micrographs & 569 \\
\hline Initial particle images (no.) & 718,578 \\
\hline Final pixel size & 0.91 \\
\hline Final particle images (no.) & 186,841 \\
\hline Number of optics groups & 400 \\
\hline FSC threshold & 0.143 \\
\hline Final map resolution $(\AA)$ & 2.8 \\
\hline Final post-processing B-Factor $\left(\AA^{2}\right)$ & -52 \\
\hline
\end{tabular}


IUCrJ (2021). 8, doi:10.1107/S2052252520013482 Supporting information, sup-7

Supplemental Table S2 - Model building statistics.

\begin{tabular}{|c|c|c|c|c|c|}
\hline Structure from Figure 4 & $4 \mathrm{~A}$ & 4B & $4 C$ & 4D & $4 E$ \\
\hline EMDB/PDB ID & 22754/7K9L & 22755/7K9X & 22756/7KA2 & 22757/7KA3 & 22758/7KA4 \\
\hline Resolution (§) & 4.9 & 3.8 & 3.6 & 3.3 & 2.8 \\
\hline Postprocessing B-Factor $\left(\AA^{2}\right)$ & -347 & -168 & -105 & -91 & -52 \\
\hline $\begin{array}{l}\text { B-Factor ( } \AA 2) \text { (Rosenthal et. } \\
\text { al. 2003) }\end{array}$ & -400.0 & -274.0 & -202.0 & -185.1 & -129.9 \\
\hline RELION Version & 3.0 .2 & 3.0 .8 & 3.0 .8 & 3.1 & 3.1 \\
\hline Bonds (RMSD) & & & & & \\
\hline Length $(\AA)$ & 0.007 & 0.008 & 0.008 & 0.004 & 0.005 \\
\hline Angles $\left(^{\circ}\right)$ & 1.197 & 0.970 & 0.973 & 0.767 & 0.894 \\
\hline Molprobity score & 2.18 & 2.21 & 1.94 & 1.35 & 1.86 \\
\hline Clash score & 9.26 & 6.36 & 6.08 & 3.99 & 4.23 \\
\hline Ramachandran plot (\%) & & & & & \\
\hline Outliers & 0 & 0 & 0 & 0 & 0 \\
\hline Allowed & 3.52 & 3.81 & 4.40 & 2.93 & 3.23 \\
\hline Favored & 96.48 & 96.19 & 95.60 & 97.07 & 96.77 \\
\hline Rotamer outliers (\%) & 3.96 & 6.12 & 2.52 & 0.36 & 3.96 \\
\hline CaBLAM outliers (\%) & 3.54 & 2.95 & 2.95 & 2.95 & 2.65 \\
\hline
\end{tabular}


IUCrJ (2021). 8, doi:10.1107/S2052252520013482 Supporting information, sup-8

\begin{tabular}{|c|c|c|c|c|c|}
\hline CC (mask) & 0.78 & 0.81 & 0.83 & 0.82 & 0.82 \\
\hline CC (box) & 0.67 & 0.79 & 0.87 & 0.76 & 0.77 \\
\hline CC (peaks) & 0.58 & 0.71 & 0.80 & 0.72 & 0.73 \\
\hline CC (volume) & 0.79 & 0.81 & 0.83 & 0.81 & 0.81 \\
\hline $\begin{array}{l}\text { RMSD }(\AA) \text { all atoms } \\
\text { compared to Figure } 4 \mathrm{E}\end{array}$ & 1.01 & 0.80 & 0.84 & 0.62 & - \\
\hline $\begin{array}{l}\text { Resolution values of map- } \\
\text { to-model FSC from } \\
\text { Supplemental Figure } 4 \text { (A) }\end{array}$ & 5.3 & 4.1 & 3.8 & 3.6 & 3.2 \\
\hline
\end{tabular}

